\title{
The LUVOIR architecture "A" coronagraph instrument
}

L. Pueyo, N. Zimmerman, M. Bolcar, T. Groff, C. Stark, et al.

L. Pueyo, N. Zimmerman, M. Bolcar, T. Groff, C. Stark, G. Ruane, J. Jewell, R. Soummer, K. St. Laurent, J. Wang, D. Redding, J. Mazoyer, K. Fogarty, Roser Juanola-Parramon, S. Domagal-Goldman, A. Roberge, O. Guyon, A. Mandell, "The LUVOIR architecture "A" coronagraph instrument," Proc. SPIE 10398, UV/Optical/IR Space Telescopes and Instruments: Innovative Technologies and Concepts VIII, 103980F (13 September 2017); doi: $10.1117 / 12.2274654$

EDent: SPIE Optical Engineering + Applications, 2017, San Diego, California, United States 


\title{
The LUVOIR Architecture "A" coronagraph instrument
}

\author{
L. Pueyo ${ }^{a}$, N. Zimmermann ${ }^{b}$, M. Bolcar ${ }^{b}$, T. Groff ${ }^{b}$, C. Stark ${ }^{a}$, G. Ruane ${ }^{c}$, J. Jewell ${ }^{d}$, R. \\ Soummer ${ }^{a}$, K. St Laurent ${ }^{a}$, J. Wang ${ }^{c}$, D. Redding ${ }^{d}$, J. Mazoyer ${ }^{e}$, K. Fogarty ${ }^{e}$, Roser \\ Juanola-Parramon ${ }^{b}$, S. Domagal-Goldman ${ }^{b}$, A. Roberge ${ }^{b}$, O. Guyon ${ }^{f}$, A. Mandell ${ }^{b}$. \\ ${ }^{a}$ Space telescope Science Institute, 3700 San Martin Drive, Baltimore, MD 21218, USA \\ ${ }^{b}$ NASA Goddard Space Flight Center, 8800 Greenbelt Rd., Greenbelt, MD, 20771 USA \\ ${ }^{c}$ Department of Astronomy, California Institute of Technology, 1200 E. California Blvd., \\ Pasadena, CA 91125, USA \\ ${ }^{d}$ Jet Propulsion Laboratory, California Institute of Technology, 4800 Oak Grove Dr., \\ Pasadena, CA 91109, USA \\ ${ }^{e}$ Department of Physics and Astronomy, Johns Hopkins University, Baltimore, 3900 Charles \\ Street, Baltimore, MD, 21230, USA \\ ${ }^{f}$ College of Optical Science, University of Arizona, 1630 E University Blvd, Tucson, AZ 85719, \\ USA
}

\begin{abstract}
In preparation for the Astro 2020 Decadal Survey NASA has commissioned the study four flagship missions spanning to a wide range of observable wavelengths: the Origins Space Telescope (OST, formerly the Far-Infrared Surveyor), Lynx (formerly the X-ray Surveyor), the Large UV/Optical/Infrared Surveyor (LUVOIR) and the Habitable Exoplanet Imager (HabEx). One of the key scientific objectives of the latter two is the detection and characterization of the earth-like planets around nearby stars using the direct imaging technique (along with a broad range of investigations regarding the architecture of and atmospheric composition exoplanetary systems using this technique). As a consequence dedicated exoplanet instruments are being studied for these mission concepts. This paper discusses the design of the coronagraph instrument for the architecture "A" (15 meters aperture) of LUVOIR. The material presented in this paper is aimed at providing an overview of the LUVOIR coronagraph instrument. It is the result of four months of discussions with various community stakeholders (scientists and technologists) regarding the instrument's basic parameters followed by meticulous design work by the the GSFC Instrument Design Laboratory team. In the first section we review the main science drivers, presents the overall parameters of the instrument (general architecture and backend instrument) and delve into the details of the currently envisioned coronagraph masks along with a description of the wavefront control architecture. Throughout the manuscript we describe the trades we made during the design process. Because the vocation of this study is to provide a baseline design for the most ambitious earth-like finding instrument that could be possibly launched into the 2030's, we have designed an complex system privileged that meets the ambitious science goals out team was chartered by the LUVOIR STDT exoplanet Working Group. However in an effort to minimize technological risk we tried to maximize the number of technologies that will be matured by the WFIRST coronagraph instruments.
\end{abstract}

Keywords: planetary systems - techniques: coronagraphy, wavefront control

\section{INTRODUCTION}

In preparation for the Astro 2020 Decadal Survey NASA has commissioned the study of four flagship missions spanning to a wide range of observable wavelengths: the Origins Space Telescope (OST, formerly the Far-Infrared Surveyor), Lynx (formerly the X-ray Surveyor), the Large UV/Optical/Infrared Surveyor (LUVOIR) and the Habitable Exoplanet Imager (HabEx). One of the key scientific objectives of the latter two is the detection and

Further author information: (Send correspondence to Laurent Pueyo) Laurent Pueyo: E-mail: pueyo@stsci.edu

UV/Optical/IR Space Telescopes and Instruments: Innovative Technologies and Concepts VIII edited by Howard A. MacEwen, James B. Breckinridge, Proc. of SPIE Vol. 10398, 103980F (c) 2017 SPIE $\cdot$ CCC code: 0277-786X/17/\$18 · doi: 10.1117/12.2274654 

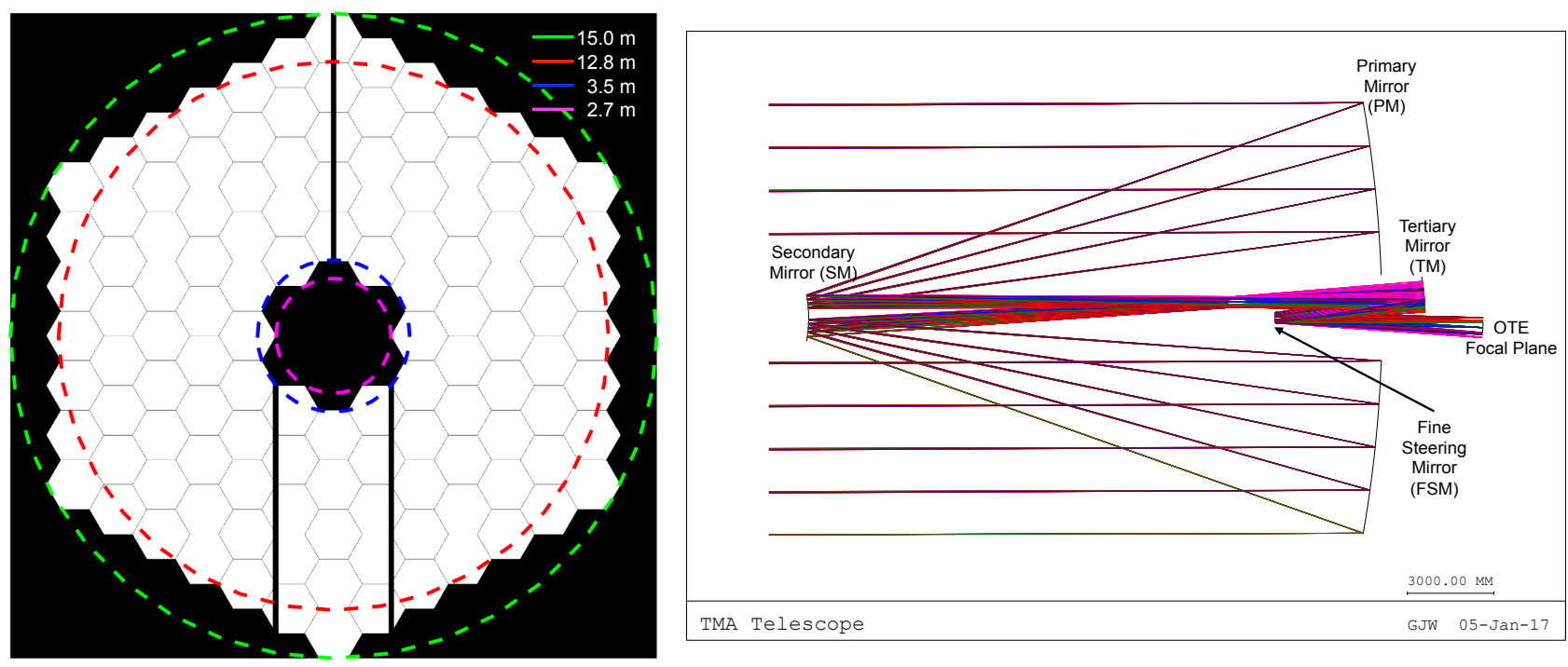

Figure 1: Left: Aperture geometry of the LUVOIR Architecture "A" pupil. Right: Optical design of the telescope. The instruments are located behind the primary, in the Backplane Support Frame. Courtesy of the GSFC Integrated Design Laboratory.

characterization of the earth-like planets around nearby stars using the direct imaging technique (along with a broad range of investigations regarding the architecture of and atmospheric composition of exoplanetary systems using this technique). As a consequence dedicated exoplanet instruments are being studied for these mission concepts. This paper discusses the design of the coronagraph instrument for the architecture "A" of the Large Ultra Violet Optical and near Infra Red Surveyor. The LUVOIR STDT (Science and Technology Definition team) has decided to put forth for evaluation two mission architectures that bracket a range of options of varying capability, cost, and risk. Architecture A is the larger of these two concepts, with a 15-m diameter primary mirror that maximizes science yield while accepting moderate technical and programmatic risk. The coronagraph instrument is capable of providing starlight suppression levels of ten orders of magnitude in multiple bandpasses over a broad range of wavelengths in order to detect and characterize the light reflected from potential earth-twins. It has has been designed to accommodate for the segmented geometry of the LUVOIR aperture "A "telescope design, discussed in the present proceedings by Bolcar el al. ${ }^{1}$ As shown in Fig. 1 the aperture is composed of 5 rings of $1.15 \mathrm{~m}$ reflective segments, separated by $6 \mathrm{~mm}$, with a central obscuration covering the equivalent area of the inner two rings, for a total of 120 active segments. The segment's actuation architecture and the envisioned basic alignment and commissioning procedures are very similar to one implemented on JWST, ${ }^{2}$ albeit with a larger number of degrees of freedom. However in order to deliver the exquisite wavefront stability required for exo-earth imaging the LUVOIR team is studying the addition of edge sensors along with an extra fine mechanisms with picometer resolution on each actuator. These features are described in more detail in Feinberg et al., ${ }^{3}$ and will be discussed in the wavefront control sub-section of this manuscript.

The material presented in this paper is aimed at providing an overview of the LUVOIR coronagraph instrument. It is the result of four months of discussions with various community stakeholders (scientists and technologists) regarding the instrument's basic parameters, followed by meticulous design work by the the GSFC Instrument Design Laboratory team. In the first section we briefly review the main science drivers and emphasize those that are the most stressing on the coronagraph design. Section two then presents the overall parameters of the instrument (general architecture and back-end instrument). Finally in section three we delve into the details of the coronagraph masks, which will have the most significant impact on the total yield of the mission, along with a description of the wavefront control architecture, which will provide the exquisite wavefront stability necessary to the imaging of exo-earths. 


\section{EXOPLANETARY SCIENCE ENABLED BY LUVOIR ARCHITECTURE A}

The LUVOIR architecture "A" corresponds to the largest aperture that can be packed into a NASAs SLS Block 2 heavy lift vehicle. Because of this large aperture, it is the most ambitious exo-earth imaging platform studied in detail by NASA to date. As a consequence, the scientific goals of the coronagraph instrument are commensurate with the ambitious philosophy underlying architecture "A". They are organized around two key science themes: a) measuring the occurrence rate of biomarkers in the atmosphere of rocky planets orbiting in the Habitable Zone (HZ) of their host stars, b) studying the broader diversity of exo-planetary systems (giant planets, circumstellar disks). The former scientific theme is significantly more stressing on the instrument and is the driver underlying the design decisions for LUVOIR architecture "A" coronagraph presented herein. As discussed in Stark et al. ${ }^{4}$ any mission aimed at measuring the occurrence rate of biomarkers in the atmosphere of nearby HZ rocky planets, ought to first be capable of detecting a statically significant ensemble of exo-earth candidates. The detectability of exoplanets in long coronagraph exposures depends on both the level of contrast achieved in the high-contrast region of the focal plane (Dark Hole, DH) and on the off-axis throughput of the coronagraph at the apparent separation of the planets, as discussed in Ruane et al. ${ }^{5}$ This is often expressed using the Inner and Outer Working Angles (IWA and OWA) scalar metrics. There are two possible definitions for IWA and OWA: the quantities that describe the inner and outer edge of the of the instrument's $\mathrm{DH}, I W A_{D H}, O W A_{D H}$, and the more astrophysically motivated definition, $I W A_{\text {planet }}$ and $O W A_{\text {planet }}$, that correspond to the star-planet separations between which the transmissivity of the planet signal is "sufficient enough". As shown on Fig. 2, the distribution of the inner and outer edge of the HZ for a notional LUVOIR architecture "A" target list (based on Stark et al. ${ }^{4}$ ) is a smooth histogram, as a consequence designing coronagraph using hard $I W A_{\text {planet }}$ and $O W A_{\text {planet }}$ metrics does not capture the full complexity of the yield optimization problem at hand. A more comprehensive approach would design a coronagraph instrument that maximizes the projected "habitable volume" integrated over the target list. This process would ideally entail an optimization of the coronagraph design parameters that includes priors on the HZ boundaries for each source in the target list and assumption on planet albedo and phase functions. This exercise would be a de-facto inversion of the yield calculations described in Start et al. ${ }^{6}$ This is beyond the scope of the LUVOIR study and in practice we work following broad parameters:

- The exo-earth detection will be carried out at visible wavelengths (in this paper we are using $600 \mathrm{~nm}$
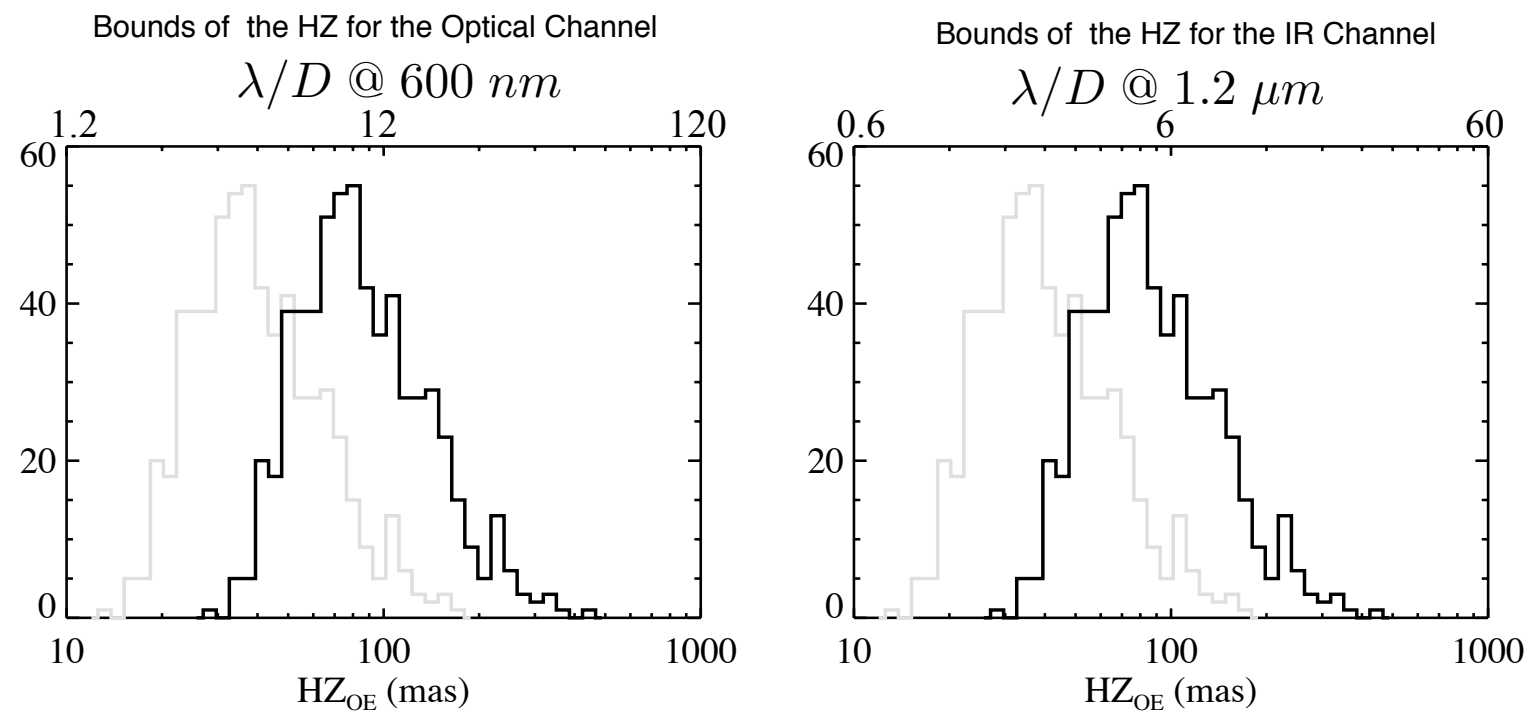

Figure 2: Histogram of the inner (grey) and outer (black) edges of the Habitable Zone on each star of a national LUVOIR target list based on Stark et al. ${ }^{4}$ Because of the wide wavelength range of the LUVOIR Architecture " $A$ " coronagraph instrument this drives coronagraph to a wide range of $I W A_{D H}$ and $O W A_{D H}$ depending on the wavelength of observations. 
as a the baseline wavelength for detection). At this wavelength, we design coronagraphs with starlight suppressions smaller than $10^{-10}$ in a region between $I W A_{D H}$ and $O W A_{D H}$ that encompasses at least $75 \%$ of the HZs real estate defined in Fig. 2.

- Instead of designing coronagraphs using hard values of $I W A_{\text {planet }}$ and $O W A_{\text {planet }}$, we seek to maximize the integrated off-axis throughput between $I W A_{D H}$ and $O W A_{D H}$ at visible wavelengths.

- We also seek to maximize the wavelength coverage, from the UV to the near IR, in order to characterize as thoroughly as possible the detected exoplanets. To do so we consider a suite of coronagraph designs whose parameters are tuned as a function of the observing wavelengths. We empirically vary these parameter in order to maximize the area covered by cross section of DHs all wavelengths. Note that using this strategy only a fraction of the exoplanets detected in the visible will be characterized in across the full wavelength range of the instrument.

At this stage of our study, we have produced a set of representative coronagraph designs following these general guidelines and we are in the process of assessing their yields. We expect to refine this strategy and refine some of the design parameters as a result of the our careful assessment of the yield of each solution.

The characterization of identified exo-earth candidates is equally as important as their detection. Fig. 3 shows a simulated spectrum of a mature earth, along with an 2 Gyrs old Archean earth, generated with the LUVOIR STDT online exoplanet spectrum simulation tool ${ }^{*}$, using the underlying models by Arney et al. ${ }^{7}$ and Robinson. ${ }^{8}$ This example illustrates the most salient characteristics of the atmosphere of earth analogs which we seek to characterize with great precision using the LUVOIR architecture "A" coronagraph. These translate into the following three requirements on the back end spectrograph: (1) Continuous spectral coverage from 200 $\mathrm{nm}$ to $2.5 \mu \mathrm{m}$ in order to capture most spectral features associated with carbon and oxygen based molecules, (2) resolution of 30 from 200 to $400 \mathrm{~nm}$ and of at least 150 (and above 1000 if possible) in the optical and near-IR. Note that because of the telescope operating temperature discussed in Bolcar et al., ${ }^{1}$ spectroscopy of faint exo-earths beyond $1.6 \mu \mathrm{m}$ will be limited to the closest target du to thermal background. However, redder spectral coverage will be invaluable to the study in detail the atmosphere of our nearest neighbors (along with characterizing larger planets).

\section{GENERAL INSTRUMENT DESCRIPTION}

\subsection{Overview of the instrument}

The coronagraph sits in the the Backplane Support Frame (BSF) of the telescope, alongside the High Definition Imager instrument (wide-field camera). The light is directed to the coronagraph by a pick off mirror alongside via the Fine Steering Mirror located after the telescope tertiary, which feeds the beam into the instrument via at a focus, as shown in Fig. 1. The LUVOIR architecture "A" coronagraph is a complex instrument, as captured on Fig. 4. At the top level, this complexity stems from the need of having continuous spectral coverage from $200 \mathrm{~nm}$ to $2.5 \mu \mathrm{m}$. In order to accommodate for the variety of high reflectivity coatings and detector technologies that span this a large wavelength range, the instrument is split in three channels that cover the following bandpasses: UV (200 to $400 \mathrm{~nm}$ ), Optical (400 $\mathrm{nm}$ to $850 \mathrm{~nm}$ ) and Infra-Red (IR, $850 \mathrm{~nm}$ to 2.5 microns). This is achieved using a series of dichorics at the entrance of the instrument. Self-consistent atmospheric modeling across the entire spectral window of the instrument requires to design these dichorics to include wavelength overlap between two adjacent channels. The 45 degrees incidence angle associated with them will create a significant polarization signature in the wavefront that might require splitting polarization at the detector level, is a similar fashion as the WFIRST architecture does. ${ }^{9}$ This aspect has not been studied yet by the LUVOIR technology Working Group, but will be the subject of future scrutiny. Each channel is equipped with two Deformable Mirrors (DMs) for wavefront control, a suite of coronagraph masks, a Low Order Wavefront Sensor (LOWFS ${ }^{10}$ ), and separate science imagers and spectrographs. Because wavefront control can only be practically achieved over finite bandpasses (as discussed in Shaklan et al., ${ }^{11}$ and as currently demonstrated in the WFIRST testbeds ${ }^{12}$ ), each channel is split into multiple bandpasses that can be sequentially selected using a filter wheel mechanism. As a consequence, while the three channels can operate in parallel, each channel can only observe in one bandpass

\footnotetext{
${ }^{*}$ http://jt-astro.science:5106/coronmodel
} 
at a time. This has an impact on the observing efficiency. For illustration we provide on Fig. 5 the optical design of the visible channel: in addition to the DMs the moving mechanisms are pupil wheels for the coronagraph apodizers, focal plane mask, Lyot stops, and filters. The relay optics are off-axis parabolas and the design has been optimized to provide residuals of $10.5 \mathrm{~nm} \mathrm{rms}$ wavefront error in the UV, 14.4 in the Optical, and $14.1 \mathrm{in}$ the IR. Thermal zones are indicated on Fig. 4: the bulk of the instrument is at the temperature of the telescope, $270 \mathrm{~K}$, to the notable exception of the UV and Optical detectors that are cooled at $170 \mathrm{~K}$, and the IR detector at $70 \mathrm{~K}$. This choice of thermal zones corresponds to a trade between system complexity (more cold optics) and redder wavelength coverage. In the absence of a cooled primary mirror we found that the gain in sensitivity at longer wavelengths (e.g. reducing the error bars at wavelengths longer than $1.6 \mu \mathrm{m}$ in Fig. 3) was small compared to the increased system complexity. A passive cooling system has been designed to accommodate these thermal zones. The thermal architecture of the observatory is described in Park et al. ${ }^{13}$ We now discuss the instrument features whose complexity has been driven by the LUVOIR Architecture "A" ambitious since goals: detector technology, back end instrument architecture, and mechanisms. In the next section we will discuss the specifics associated with coronagraph mask design and wavefront control.
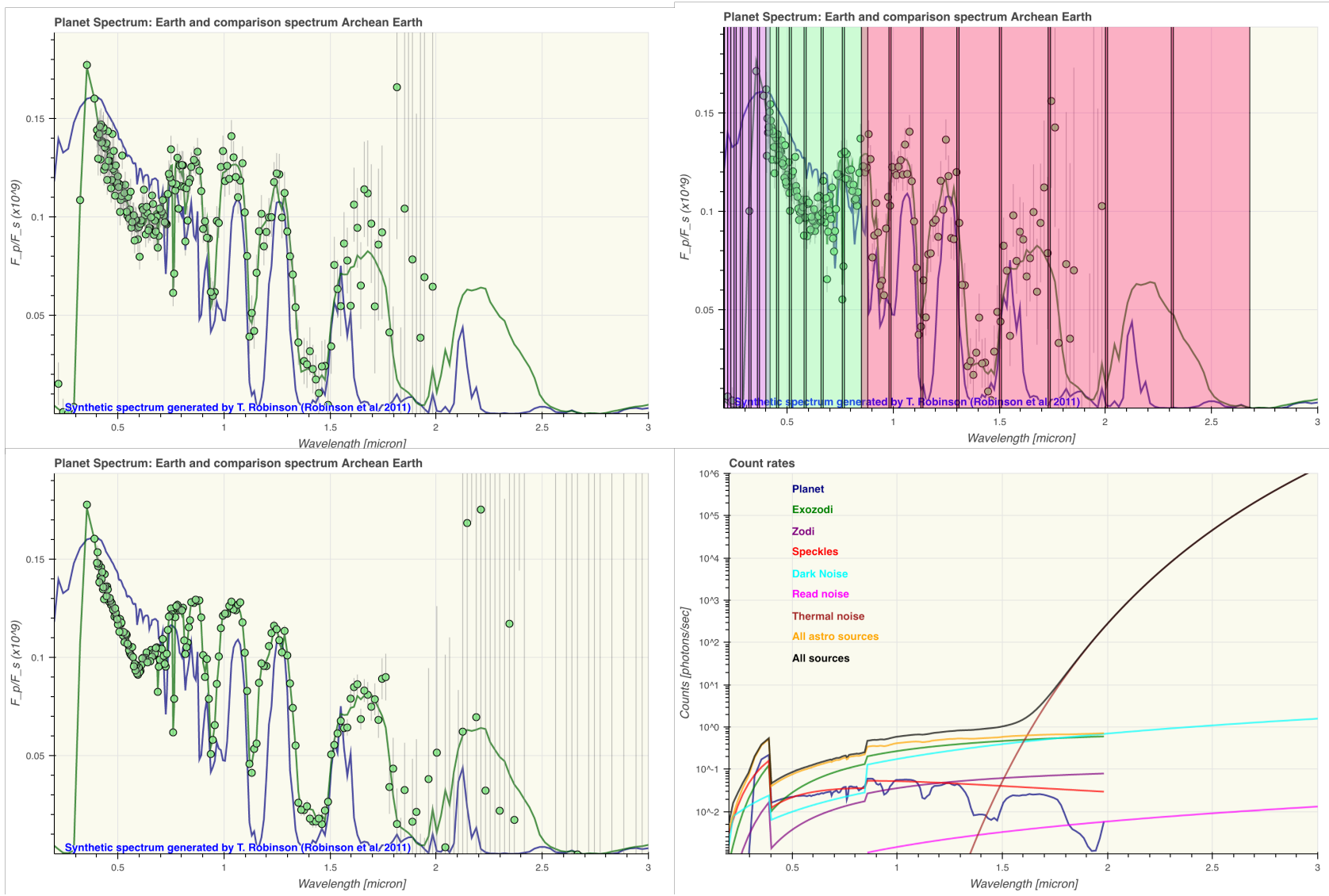

Figure 3: Simulations of the spectrum of an earth-like planet seen by the LUVOIR Architecture A coronagraph for an exposure time of 24 hrs per bandpass. Top Left: earth-like planet at 1 AU around a sun at 15 pc. Bottom Left: earth-like planet at 1 AU around a sun at 7 pc. In both cases the data quality is sufficient to identify molecular absorptions that enable to ambiguously discriminate a mature earth from and archean earth. ${ }^{7,8}$ Top Right: The bandpasses in each channel of the LUVOIR coronagraph instrument defined in this manuscript are overlayed to the spectrum. UV, Optical and IR channels can operate in parallel, however the bandpasses in each channel can only be operated sequentially. Thus the observing time to obtain this spectrum would be of the order of a week. Bottom Right: wavelength dependent noise budget associated with the 15 pc simulation. Longward of $1.6 \mu \mathrm{m}$ the field thermal noise overwhelms the planet signal. These calculations were carried out with the LUVOIR exoplanet spectrum online tool: http://jt-astro.science:5106/coronmodel 

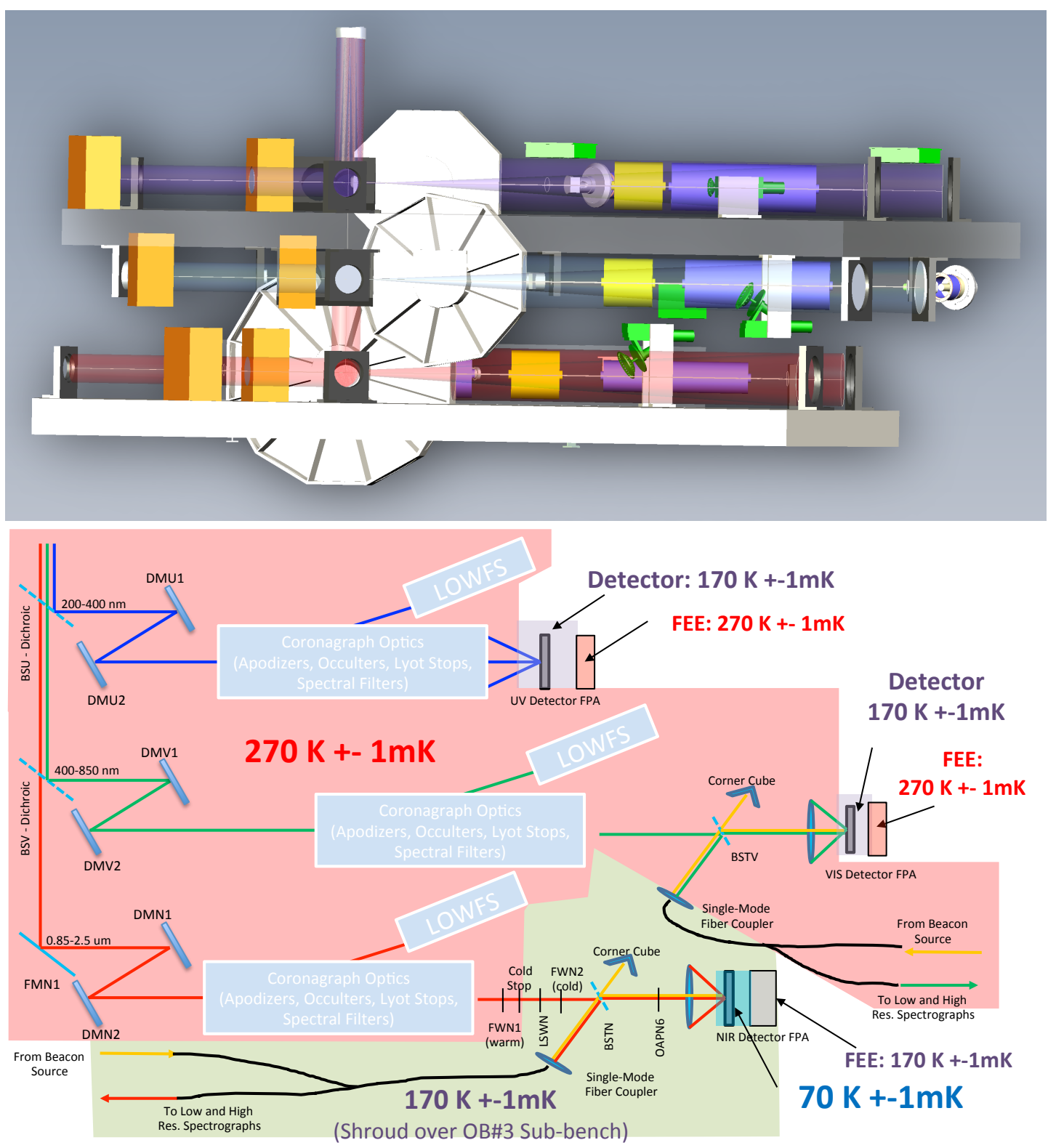

Figure 4: Top: Mechanical model of the LUVOIR Architecture "A" coronagraph instrument. The pupil and focus wheel mechanisms for the three channels (UV, Optical IR) are the main volume drivers. : Bottom: System level diagram of the LUVOIR Architecture "A" coronagraph instrument identifying the three channels along with thermal zones. Courtesy of the GSFC Instrument Design Laboratory team.

\subsection{Detectors}

The detector technologies are specific to each channel and our baseline choices heavily rely on technology that has been used on previous missions or planned in the WFIRST baseline instruments:

- Optical: the baseline technology for the visible $(400-850 \mathrm{~nm})$ channel is an e2v EMCCD, with a format of $1024 \times 1024,13 \mu \mathrm{m}$ pixels. This technology is based on a silicon CCD with low-noise, on-chip, multiplication gain register (1000 times). It is the baseline detector for the WFIRST coronagraph instruments. We assumed zero read noise (in photon-counting mode) and a median Dark Current of $1 \mathrm{e}-4 \mathrm{e}-/ \mathrm{pix} / \mathrm{sec}$ (as measure by JPL WFIRST project ${ }^{14,15}$ ) for our radiometry calculations. 
- Near-IR: the baseline technology for the near-IR is a Teledyne HAWAII 4RG with SIDECAR ASIC, with a format of $4096 \times 4096,13 \mu \mathrm{m}$ pixels. This technology HgCdTe detectors, indium bump-bonded to a CMOS circuit, with SIDECAR ASIC Focal Plane Electronics. It is the baseline detector for the WFIRST wide-field instrument as well as JWST-NIRCam. We assumed a median read noise ¡10 e-/pix at $100 \mathrm{kHz}$ and 77K, and a Median Dark Current: 2e-3 e-/pix/sec (measured 90-110K) for our radiometry calculations.

- UV : the baseline technology is similar to the Visible channel except that the detector surface has been $\delta$-doped processed. We assumed the same characteristic as the Visible channel detector, however this technology is not fully mature yet and requires some investment.

\subsection{Back end imagers spectrographs}

Each channel will be equipped with an imaging detector of the technology that is described above. Spectroscopy is not required at UV wavelengths and the imager (field of view square 1.4" $\times 1.4$ " squared) is the only science detector. Both an imager and spectrograph are required for the Optical and the IR channel. The respective fields of view of the imagers are 2.7" $\times 2.7 "$ and 5.6" $\times 5.6 "$. These sizes are larger than the angular extend of the HZ shown in Fig. 2 in order to enable the detection and characterization of outer planets in the exo-solar systems detected (either using coronagraphs with $O W A_{D H}$ much larger than the outer edge of the HZ, or for giant planets at separations much larger than $O W A_{D H}$ ). For the Optical and IR spectrographs we first studied the feasibility of Integral Field Spectrograph (IFS) such as the ones installed on ground based instruments ${ }^{16-19}$ and the one envisioned for WFIRST. ${ }^{20}$ All post coronagraph IFS require to Nyquist sample the instrument Point Spread Function (PSF), which means having at least two spatial elements per unit of the angular resolution (two pixels per $\lambda / D)$. Assuming a design similar to the one of the Gemini Planet Imager instrument (GPI), field of view of $\sim 2.7$ ", $\mathrm{R} \sim 50$, Nyquist sampled at $\sim 1.2$ microns on a 8 meters telescope with a 2048 squared detector, we can derive to the approximate dimensions of an IFS associated with the LUVOIR architecture "A" coronagraph. Scaling up the GPI numbers to a $15 \mathrm{~m}$ meters aperture, Nyquist sampling at $\sim 600 \mathrm{~nm}$ and a resolution of $\mathrm{R} \sim$ 150, we find that Optical channel IFS would have to rely on a $10000 \times 10000$ detector, a factor of 10 beyond the EMCDD technology that will be matured by WFIRST. This prompted us to also consider a high-resolution Fiber Fed Spectrograph ${ }^{21}$ (hi-res FFS). While this solution is less mature, it presents the advantage of being a lot more efficient in terms of detector real estate, and can potentially yield higher resolution spectra for the brighter planets/most nearby systems ( $\sim 1500$ would require a 100K squared detector in the IFS configuration). We list the pros- and cons of each technique below:

- IFS pros: This technology will be TRL9 post-WFIRST. It is an intrinsically multiplexed design. That is the spectrum of multiple planets (or background sources) can be obtained at the same time, which makes the observations more efficient. The continuum of the planets spectra, essential to measure absolute molecular abundances, is preserved.

- IFS cons: Technology development needs to occur to mature low noise 10K squared visible detectors. Alternatively the field of view of the IFS could be reduced to the only encompass the HZs (dividing the field of view of the Optical channel by a factor of 3 would capture most of the HZs on Fig. 1), however this solution make the characterization of outer planets impossible. Obtaining resolutions of $\sim 1500$, an option that has been deemed preferable by the LUVOIR STDT exoplanetary science Working Group, seems hard to achieve in the IFS configuration, even with 20 year of vigorous detector technology development.

- hi-res FFS pros: Detector real estate does not limit spectral resolution. Both coherent starlight suppression (via fiber injection ${ }^{21}$ ) and post-processing gains using cross correlation with spectral templates ${ }^{22,23}$ are possible with this architecture. This potentially relaxes the requirements on the wavefront control system. This technique will be tested in multiple ground based observatories over the next decade. ${ }^{21,24,25}$ Moreover, should the observatory feature a mid to high resolution "facility" spectrograph (to be studied the LUVOIR architecture "B"), this instrument can be readily fed by a fiber picking of the planet light in the focal plane of the coronagraph instrument, instead of a having a dedicated spectrograph in the coronagraph channel.

- hi-res FFS cons: Even if ground based projects are successful, there is not path to date bring this technology to high TRL levels in the context of a space mission. The cross correlation technique cannot measure absolute molecular abundances and achromatic fiber injection needs to be demonstrated in order 


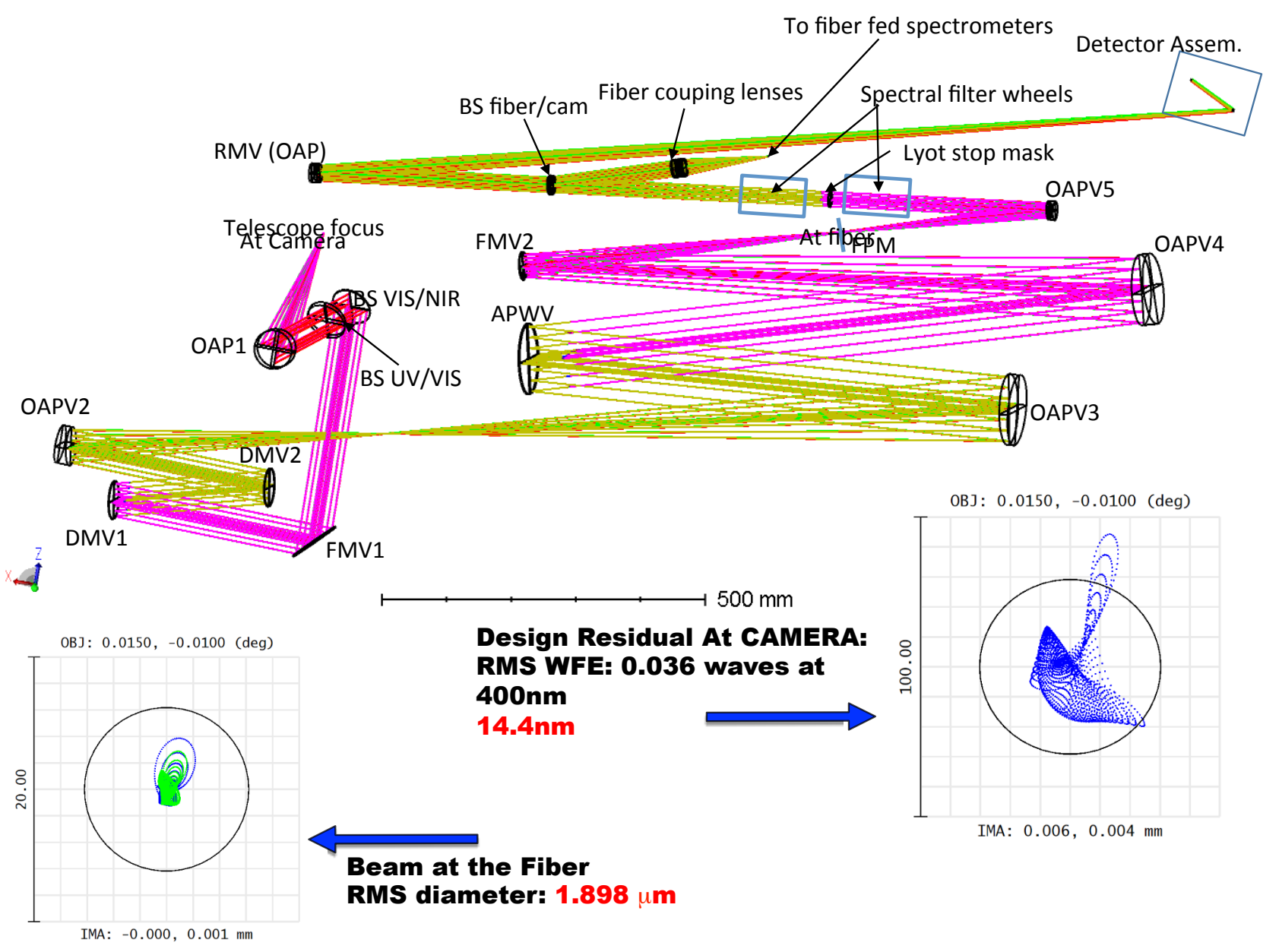

Figure 5: Optical design of the LUVOIR Architecture "A" coronagraph Optical channel. Courtesy of the GSFC Instrument Design Laboratory team.

to confidently measure the continuum of the planet's spectra. Because the spectrum of only one object at a time can be obtained, the observing efficiency of this design is inferior to the one of the IFS.

Based on these trades, the choice of a hi-res FFS as the primary spectrograph for the exoplanet imaging of LUVOIR architecture "A" was mostly driven by scientific considerations. It was the only design that could obtain both low an high resolution of earth-analogs, along with being able to characterize a wide variety of other type of planets (including outer planets). However, in the absence of a Design Reference Mission (DRM) at this stage, it is hard to quantify the impact the lack of multiplexing of this technique will have on the final exo-earths yield. In particular, the loss of efficiency associated with the inability to spectroscopically vet point source candidates without multiple follow up observations (in order to establish physical association) might have a significant impact on the overall yield. As a consequence the IFS solution is still considered as a back-up for architecture "A" until the LUVOIR STDT carries out the full architecture "A" DRM. In any case the IFS solution will be studied for architecture "B" (a smaller, $\sim 8 \mathrm{~m}$ aperture is less demanding in terms of detector real estate), and, pending on the Design Reference Mission (DRM) results, might be added to Architecture A at that point.

\subsection{Mechanisms}

Mechanisms and moving parts are a big source of system complexity and cost in space based instrument. ${ }^{26}$ The key science enabling mechanisms are: 
- Deformable Mirrors: our choice of DM technology is based on the need of high-density devices necessary to achieve large Outer Working Angles. For instance achieving high contrast for the outer region of the $\mathrm{HZ}$ around the most nearby stars requires an $O W A_{D H}$ of $\sim 48 \lambda / D$, which translates into 96 actuators across the pupil. Imaging of outer giant planets brings this number to 128 actuators. In order to keep the optical design compact we baselined Boston Micro-Machines DMs ${ }^{27}$ that feature highest density per unit length actuators on the market to date. A secondary driven was the the fact that such compact DMs result in small Fresnel numbers designs, and thus are more amenable to DM based amplitude modulation techniques such as ACAD. ${ }^{28}$ While this technology will not be matured by WFIRST at the component level, there are avenues to do so using smaller satellites. ${ }^{29,30}$

- Wheels: each channel (UV, Optical and IR ) of the instrument is equipped with a series of wheels that accommodate the various masks and filters necessary for coronagraphy: pupil wheel (for apodizers), focal plane wheel (for focal plane masks/occulters), Lyot wheel (for Lyot stop) and filter wheel (for the narrowband filters (2\% necessary for wavefront sensing) and the medium band $15 \%$ for the science. Fig. 6 summarizes how these wheels are populated for the Optical channel. The number of slots on these wheels, and thus the overall volume of the instrument, stems from the fact that for wavefront control purposes the channel has to be split into a series of $15 \%$ bands (this number was chosen based on the current state of the at associated with the WFIRST testbed demonstrations ${ }^{12}$ ) along with our choice of baseline coronagraph technology, which needs specific Apodizer + Focal Mask + Lyot Stop combination for each bandpass. Because of the significant wavelength coverage of both the Optical and IR channels (450 nm and 1.65 microns bandpass respectively) we were able to eliminate some redundancies in the mask combinations, however the number of wheel slots in each channel remains considerable. The LUVOIR STDT is now focusing on upgrading the baseline coronagraph design to reduce this complexity. Moreover should progress in broadband wavefront control occur (WFIRST recently remonstrated $18 \%$ bandpass), the system complexity will also be reduced.

- Other mechanisms: each channel is equipped with focus mechanisms. The Optical and Near-IR channels have an actuated pick off corner cube within the Fiber Injection Unit in order to steer the planet light into the point spectrograph, as described in Mawet et al. ${ }^{21}$

\section{STARLIGHT SUPPRESSION SYSTEM}

\subsection{Coronagraph design, static response}

As discussed in $\S 1$ the key driver for the LUVOIR architecture "A" coronagraph is a contrast of $10^{-10}$ in regions of the image plane that can over most of the HZ for an majority of the target stars. We studied two possible solutions for coronagraph masks: a conservative design that is robust to misalignments, stellar angular size and whose mask technology will be matured by WFIRST, the binary apodized APLC, ${ }^{31-33}$ along with a more aggressive design which will improve science performance but is less robust and mature. ${ }^{34,35}$ We describe each coronagraph mask configurations for the Optical channel, in which the search will occur, and then discuss how to extend them to the UV and IR channels.

\subsubsection{Apodized Pupil Lyot Coronagraph, Optical channel}

We used the design philosophy presented in Riggs et al. ${ }^{36}$ Zimmerman et al. $^{33}$ in the case of WFIRST, and N'Diaye et al., ${ }^{32}$ in the case of segmented apertures. We set the geometry of the focal plane mask $\left(I W A_{D H}\right.$ and $O W A_{D H}$ ), the size of the Lyot Stop and use a linear optimization to generate binary apodizers. We set the inner edge of the DH hole to be smaller than the outer edge of the focal plane makes in order to make the design robust to stellar angular size and misalignments. ${ }^{37}$ Because it is hard to design and apodizer with sufficient throughput that covers the entire possible HZ illustrated on Fig. 2, we designed coronagraph for a suite of focal plane masks, with varying $I W A_{D H}$ and $O W A_{D H}$, assuming that the masks will be chosen as a function of the distance to each observed host star ( Fig. 6) described the entire family of masks). We also assume an input pupil with larger segment gaps and secondary struts than the actual telescope design, in order to include robustness to pupil misalignments. We carry out this optimization of each one of the focal plane mask geometry 


\begin{tabular}{|c|c|c|c|c|}
\hline \multicolumn{5}{|c|}{ VIS CHANNEL APODIZING MASK LIST } \\
\hline ID & IWA at $400 \mathrm{~nm}$ & OWA at $400 \mathrm{~nm}$ & IWA at $850 \mathrm{~nm}$ & OWA at $850 \mathrm{~nm}$ \\
\hline VIS-A1 & & & 3.75 & 15 \\
\hline VIS-A2 & & & 5 & 20 \\
\hline VIS-A3 & 3.75 & 12.7 & 7 & 27 \\
\hline VIS-A4 & 4.2 & 16.5 & 9 & 35 \\
\hline VIS-A5 & 5.6 & 30.1 & 12 & 64 \\
\hline VIS-A6 & 9 & 35 & & \\
\hline VIS-A7 & 12 & 64 & & \\
\hline \multicolumn{5}{|l|}{ VIS-V1 } \\
\hline \multicolumn{5}{|l|}{ VIS-V2 } \\
\hline \multicolumn{2}{|c|}{ VIS Channel Filter wheel } & \multicolumn{3}{|c|}{ VIS Channel Focal wheel } \\
\hline \multicolumn{2}{|c|}{$400-460$ (15\% VIS Band 1$)$} & \multicolumn{2}{|c|}{ ZWFS Mirror } & \\
\hline \multicolumn{2}{|c|}{ 450-520 (15\% VIS Band 2) } & \multicolumn{2}{|c|}{ VVS Vis1 Mask 400-460 Band } & \\
\hline \multicolumn{2}{|c|}{$510-590$ (15\% VIS Band 3$)$} & \multicolumn{2}{|c|}{ VVS Vis1 Mask 450-520 Band } & \\
\hline \multicolumn{2}{|c|}{$580-670$ (15\% VIS Band 4) } & \multicolumn{2}{|c|}{ VVS Vis2 Mask 580-670 Band } & \\
\hline \multicolumn{2}{|c|}{$660-770(15 \%$ VIS Band 5$)$} & \multicolumn{2}{|c|}{$\begin{array}{l}\text { VVS Vis2 Mask } 660-770 \text { Band } \\
\text { VVS Vis2 Mask } 760-880 \text { Band }\end{array}$} & \\
\hline $760-880(15 \%$ VIS Band 6$)$ & 760-880 (15\% VIS Band 6) & \multicolumn{2}{|c|}{ APLC Vis OE 1} & \\
\hline \multicolumn{2}{|c|}{$2 \%$ WFS Filters for VIS Band 1 ( $3 x$ ) } & \multicolumn{2}{|c|}{ APLC Vis OE 2} & \\
\hline \multicolumn{2}{|c|}{$2 \%$ WFS Filters for VIS Band $2(3 x)$} & \multicolumn{2}{|c|}{ APLC Vis OE 3} & \\
\hline \multicolumn{2}{|c|}{$2 \%$ WFS Filters for VIS Band $4(3 x)$} & \multicolumn{2}{|c|}{ APLC Vis OE 4} & \\
\hline & 5 Bana $4(3 x)$ & \multicolumn{2}{|c|}{ APLC Vis OE 5} & \\
\hline $2 \%$ WFS Filt & S Band $6(3 x)$ & APLC Vis O & & \\
\hline Vis Band 10 & & APLC Vis & & \\
\hline Vis Band $1 \%$ & & APLC Vis $O$ & & \\
\hline Vis Band 0.1 & & APLC Vis O & & \\
\hline Open Slot & & APLC Vis $O$ & & \\
\hline Closed Slot & & APLC Vis 0 & & \\
\hline & & APLC Vis 0 & & \\
\hline & & APLC Vis 0 & & \\
\hline & & APLC Vis & & \\
\hline & & open & & \\
\hline TOTAL: 29 & & & & \\
\hline VIS & nel Lyot whee & & & \\
\hline Oper & & & & \\
\hline APL & Stop 1 & & & \\
\hline APL & Stop 2 & & & \\
\hline VVC & Stop & & & \\
\hline TOT & & TOTAL: 1 & & \\
\hline
\end{tabular}

Figure 6: Population of the Optical channel's Apodizer, Focal plane, Lyot and Filter wheels. These respectively have 9, 16, 4 and 24 occupied slots. The number of wheels' slots is of the same order for other channels.

in Fig. 6. Example of results are given on Figs. 7 and 8. The intrinsic throughput at a given off-axis separation (in units of $\lambda / D$ ) is measured as encircled energy in the focal plane after the coronagraph normalized by the encircled energy associated with the telescope direct Point Spread Function (PSF). It is displayed on Fig. 10: the maximum throughput is of the order of the solutions presented in N'Diaye et al. ${ }^{32}$ Note that this baseline design is however very conservative since it is capable to accommodating for a large pupil shear between the plane of the apodizer and the primary mirror. Based calculations using preliminary geometries similar to the LUVOIR Architecture "A" primary we predict that relaxing this constraint will increase the throughput by about a factor of two. 
Working angle range 4 - $9 \lambda / D, 10 \%$ bandpass, Rel. E.E.(0.7 $\lambda / D)=18.47 \%$

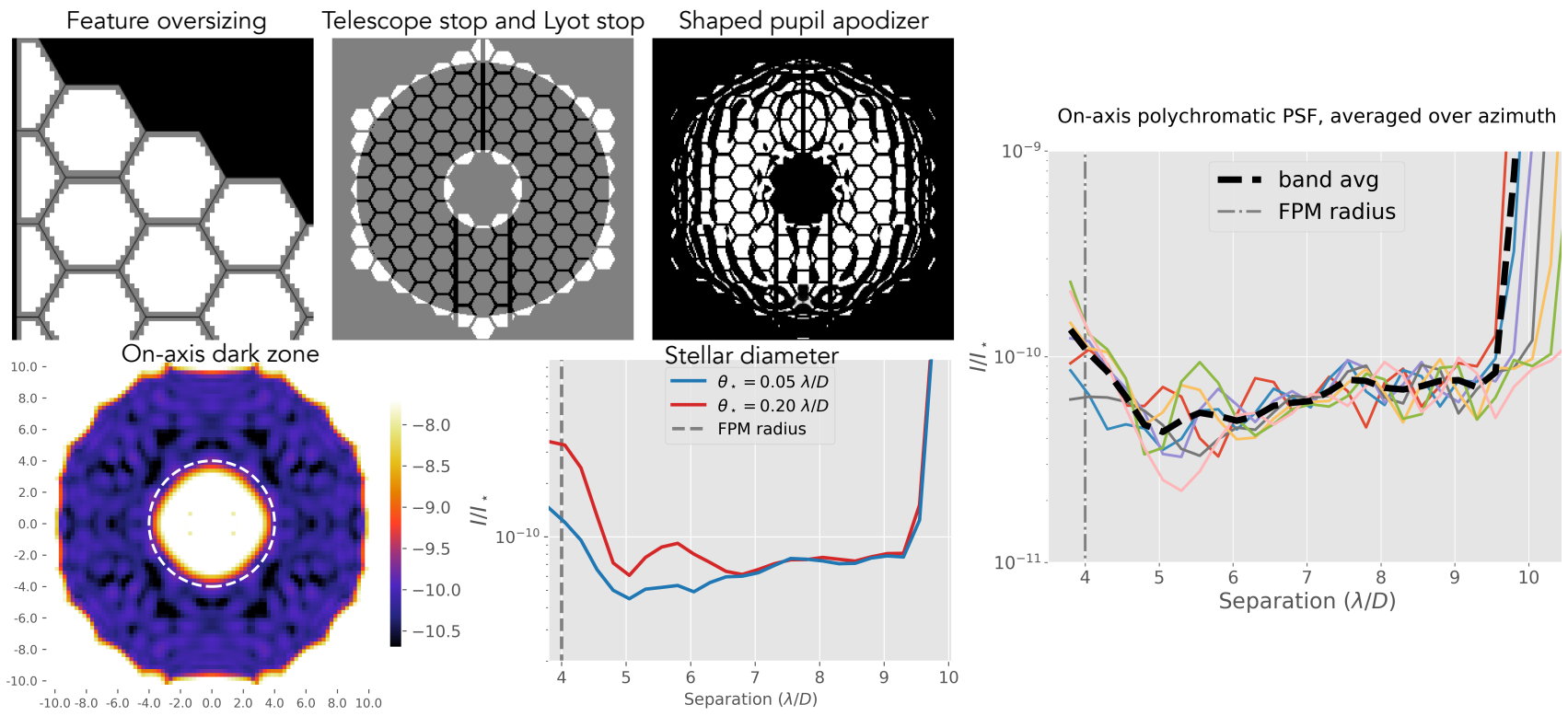

Figure 7: Coronagraph design for the LUVOIR Architecture "A" based on a hard edge focal plane mask and a binary apodizer (APLC concept). This specific focal plane mask geometry would be used to search for exo-earths in the optical around distant stars. Courtesy of N. Zimmerman.

Working angle range 8 - $24 \lambda / D, 10 \%$ bandpass, Rel. E.E.(0.7 $\lambda / D)=16.16 \%$

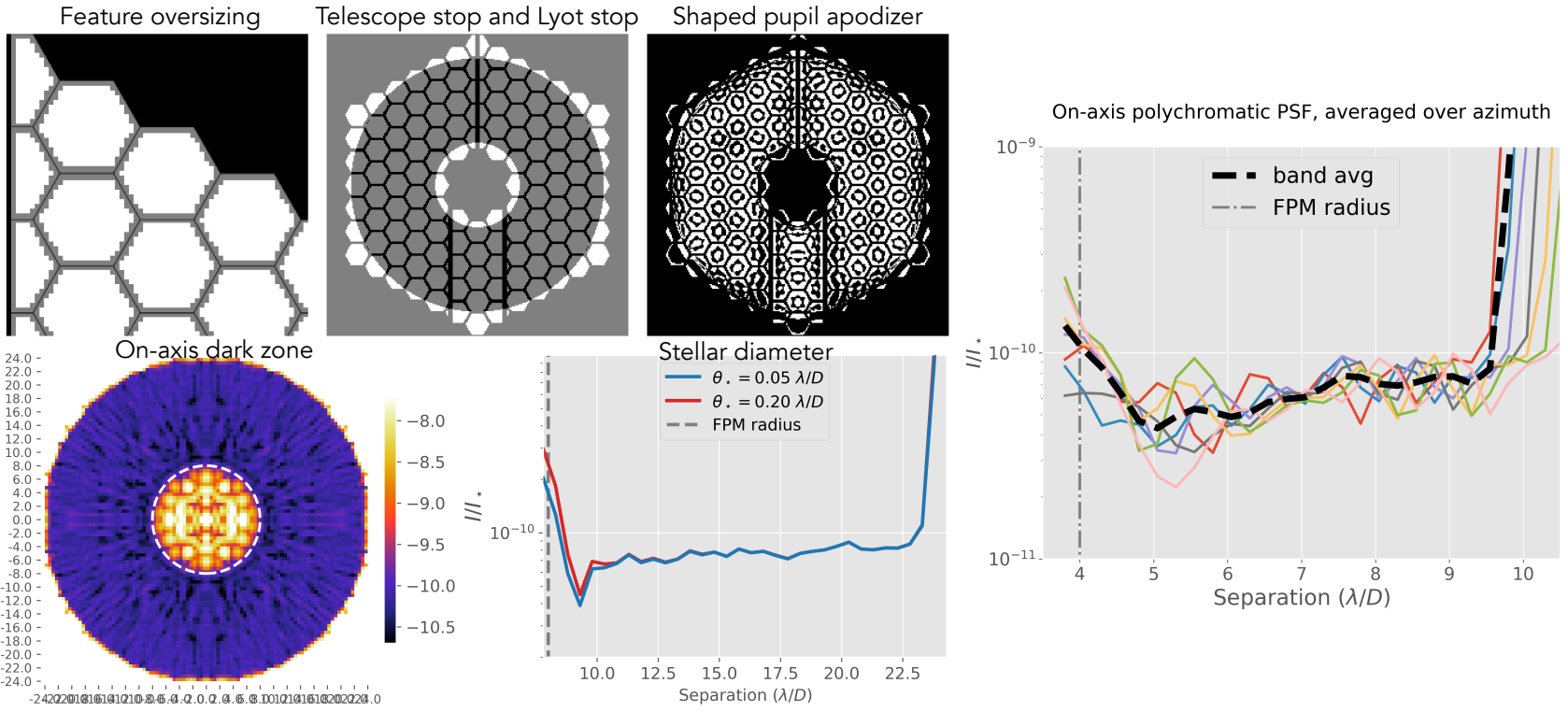

Figure 8: Coronagraph design for the LUVOIR Architecture " $A$ " based on a hard edge focal plane mask and a binary apodizer (APLC concept). This specific focal plane mask geometry would be used to search for exo-earths in the optical around the most nearby stars. Courtesy of N. Zimmerman. 


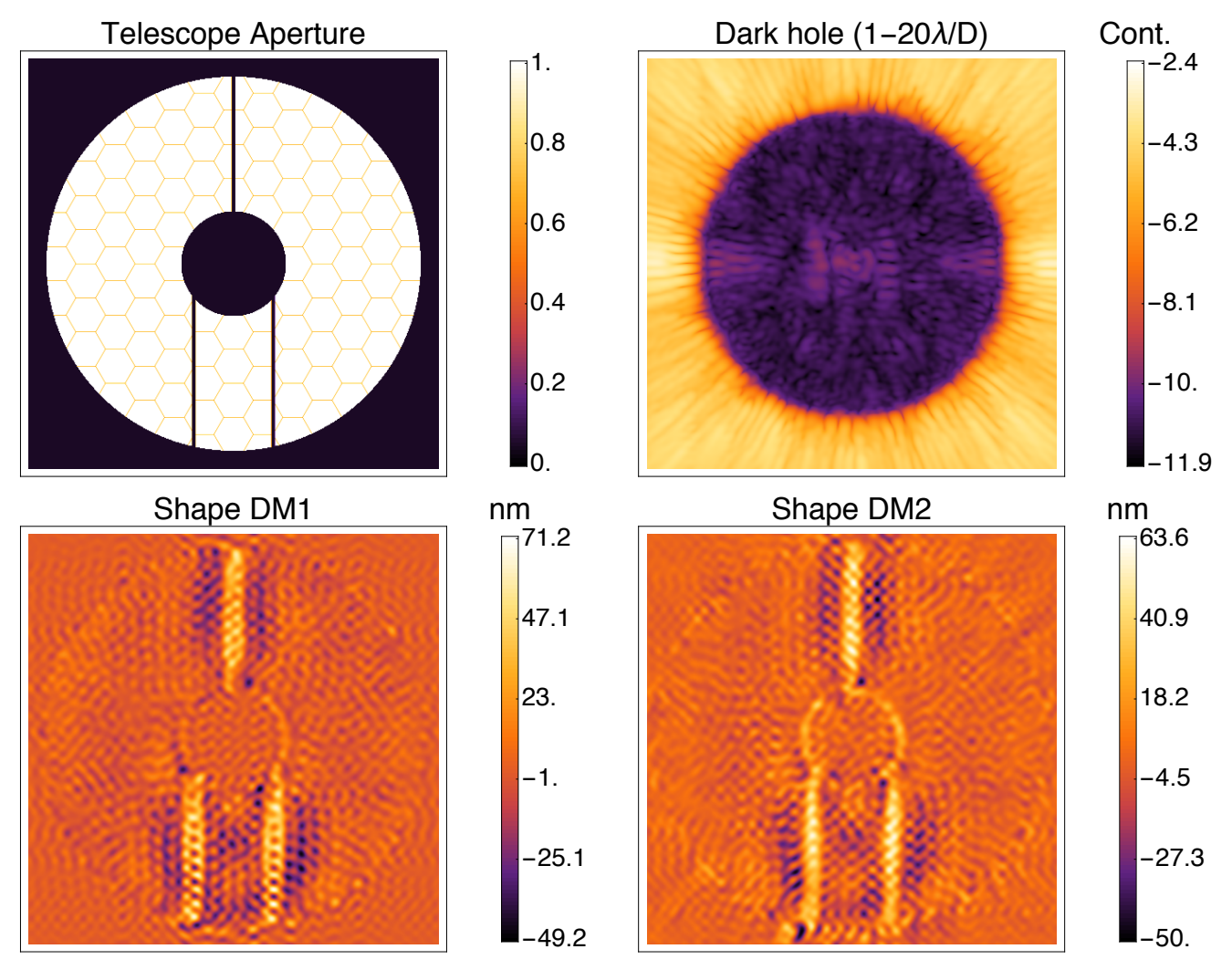

Figure 9: Coronagraph design for the LUVOIR Architecture "A" based on a Vector Vortex focal plane mask and using the ACAD-OSM technique to control the DMs. Courtesy of J. Mazoyer

\subsubsection{Vortex, Optical channel}

We combined a Vortex focal plane mask ${ }^{34,35}$ with a static grayscale apodizer in order to accommodate for the central obscuration. ${ }^{38}$ We calculate this apodizer either using an iterative optimizer which create a broadband null in the presence of the obscuration, support structures and segment gaps, ${ }^{5}$ or using polynomial functions which create a perfect null in the presence of the central obscuration. ${ }^{39}$ In this latter case, we use the two DMs (which are in the instrument for wavefront control purposes) to correct for diffraction artifacts associated with the secondary support structures and the segment gaps. ${ }^{28,40,41}$ In this paper we only show results corresponding to this latter strategy but we will evaluate yield of both methods (e.g. full grayscale apodizer and hybrid apodizer + DMs solution). Fig. 9 displays the DMs shapes and the resulting PSF with: $I W A_{D H}=1 \lambda / D$ to $O W A_{D H}=20 \lambda / D$ and a bandpass of $20 \%$. Robustness to pupil shear is not a design parameter, since the are DMs operating in closed loop and can correct for such misalignments, as discussed in Mazoyer et al. ${ }^{40}$ However, even if this design is more compelling based on the superior throughput at small separations, see Fig. 10 it suffers from two caveats when compared to the APLC. It is very sensitive to stellar angular size/low order aberrations and its flight readiness will not be improved with WFIRST. The former is a limitation of current Vortex designs on apertures with central obscuration, which do not feature the charge dependent multi-mode quasi-perfect nulling (e.g. very high starlight suppression of the first few Zernike modes as the mask topological charge increases) that exists in vortex coronagraphs on circular apertures. ${ }^{42}$ Several groups are currently investigating how to circumvent this limitation. Some of the dual DM wavefront control concepts associated with the latter will be advanced by WFIRST, but other avenues will be needed to demonstrate the flight readiness of optical vortex masks with $10^{-10}$ cancellation for LUVOIR. 


\subsubsection{Impact on performance}

The choice of coronagraph masks drives the overall scientific output of the coronagraph instrument. Because the "search of life" objective is the most challenging, we will uses it as a fiducial to assess the quality of a particular design. As discussed in $\S 1$, this yield can be expressed in two broad categories: the number of exoearth candidates detected (earth radii planets orbiting in the HZ of their host stars) under the assumption of a finite duration dedicated to this search, and how well the discovered objects get characterized (spectral coverage and uncertainties, knowledge of the orbit). Given an mission lifetime and a survey strategy (which include a fraction of the time for follow up observations), the former can be quantified using the methods described in the literature. ${ }^{6,43,44}$ Note that the latter is somewhat more qualitative since it depends on how much of the followup/characterization time is dedicated to a given system. Because detection will be carried out in the Optical channel, nearby stars will be observed using the APLC since these sources will not suffer from the relatively large $I W A_{D H}$ and benefit from the robustness to stellar angular size. For more distant stars the Vortex design will be more compelling. This is why the wheels of the instrument have ben populated with slots enabling the use of both technologies. We will first run yield calculations that assuming that the search is solely done with one single technology (either APLC or Vortex). We will then determine, based on the outcome of these calculations, the cutoff distance at which the Vortex will become more advantageous. As far as full spectral characterization is concerned: the robustness of the APLC makes it our preferred architecture for the UV channel (which is more sensitive to wavefront errors because of the shorter wavelength), and the small $I W A_{D H}$ of the Vortex makes it our preferred architecture for the Near-IR. Once our discovery yield is calculated, we will quantify in detail how many of the discovered systems can be fully characterized across the full spectral bandpass illustrated in Fig. 3. Note that the LUVOIR Architecture "A" telescope pupil has been circulated to the community: we are inviting interested parties to contribute coronagraph design that can be evaluated along the procedure outlined herein. One particularly interesting area of investigation would be to produce designs that takes advantage the full LUVOIR Architecture "A" aperture (contrarily to our baseline solutions which only uses the inscribed circle). We will revisit our wheel allocation should solution providing much higher science yield, or a similar yield but simplifying greatly the system.

\subsection{Wavefront control, dynamic response}

\subsubsection{Response of the coronagraph to wavefront errors}

The design of a high yield static coronagraph is a necessary condition to a successful exo-earth imaging instrument on the LUVOIR architecture "A" but it is not sufficient. Indeed, exquisite wavefront stability needs to occur in order for the contrast to remain at the $10^{-10}$ level throughout the duration of the long exposures that are needed for exoplanet detection and characterization. Note that the actual mapping between contrast and wavefronts stability (say in picometer rms) depends on each mode of the disturbance, and that some coronagraphs can be more robust than others to these modes. For instance, low order modes can be in the kernel of the coronagraph response (see N'Diaye et al. ${ }^{37}$ for examples with the APLC and Ruane et al. ${ }^{42}$ for examples with the Vortex). This relaxes wavefront requirements for these modes. Another example is given on Fig. 11 which illustrates the response LUVOIR architecture "A" APLC designs to segment level piston and top tip errors. Ongoing optimizations aimed at increasing the robustness of coronagraph designs to wavefront errors while maintaining high performance (high throughput at the smallest operation possible) are actively being pursued.

\subsubsection{Wavefront controlability}

Even is the coronagraph designs can be intrinsically robust to a finite amount of aberrations, they ought to be sensitive to mid-high spatial frequency aberrations since the spatial frequency of those mimics the one of an exoplanetary signal. As a consequence, each one of the three channel of the LUVOIR architecture "A' coronagraph instrument is equipped with two DMs to in order to a) shape the static wavefront to reach the adequate contrast (symmetric broadband $\mathrm{DH},{ }^{11,45-47} \mathrm{~b}$ ) to stabilize the wavefront so that high contrast is maintained throughout science exposures in spite of the presence of time varying aberrations (stemming either form thermal or mechanical disturbances). Moreover, the primary mirror can also be actuated using picometer resolution PZTs, mounted on JWST hexapod actuators, in order to stabilize the primary surface figure in the presence of mechanical disturbances (see Feinberg et al. ${ }^{3}$ for details). As a consequence the LUVOIR architecture "A "coronagraph 


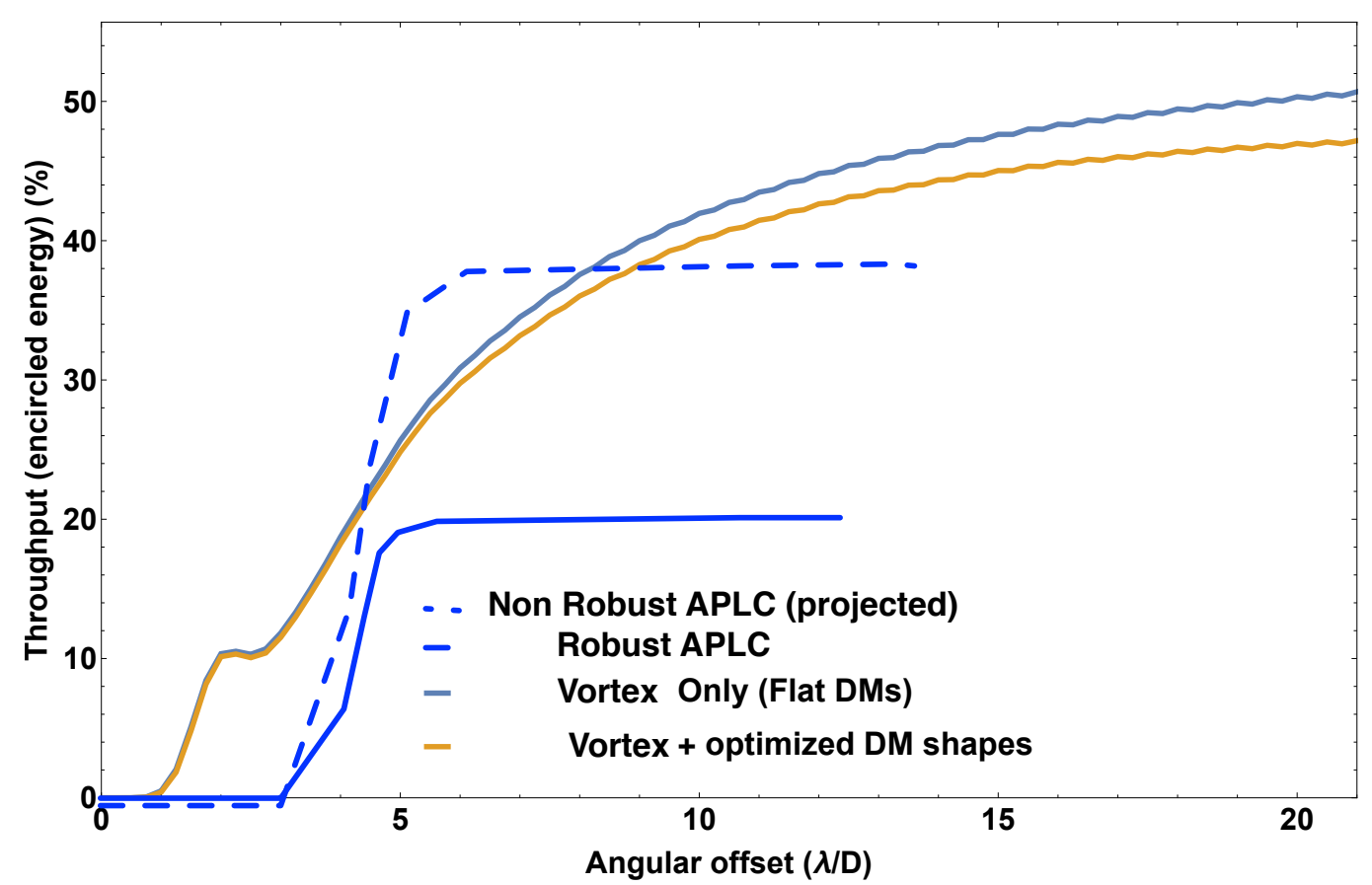

Figure 10: Throughputs as a function separations for both the small $I W A_{D H} A P L C$ and vortex designs. Note that the APLC presented herein have been designed to be robust to misalignments and low order wavefront errors. Based on previous work we can extrapolated to non robust APLC designs (not designed yet), which have a large separation throughput similar to the Vortex design, albeit with a lower one at small separation.

\section{Working angle range 4 - $9 \lambda / D, 10 \%$ bandpass, Rel. E.E.(0.7 $\lambda / D)=18.47 \%$ Segment alignment sensitivity - contrast degradation at $700 \mathrm{~nm}$}

Random per-segment pistons Normal distrib. with 10 pm std dev 50 pm P-V across aperture

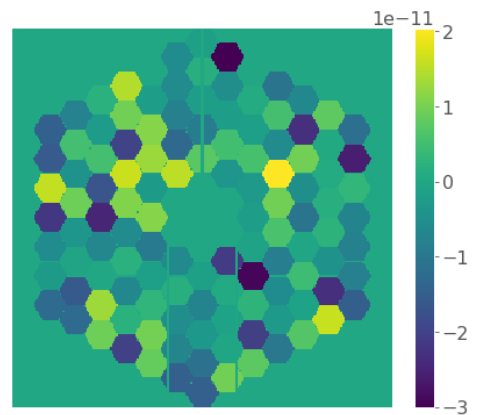

Random per-segment tip-tilts Normal distrib. with 10 pm std dev 79 pm P-V across aperture

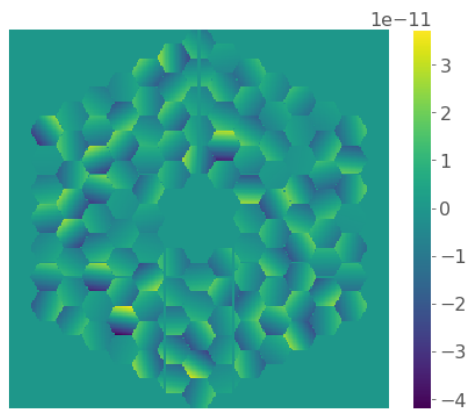

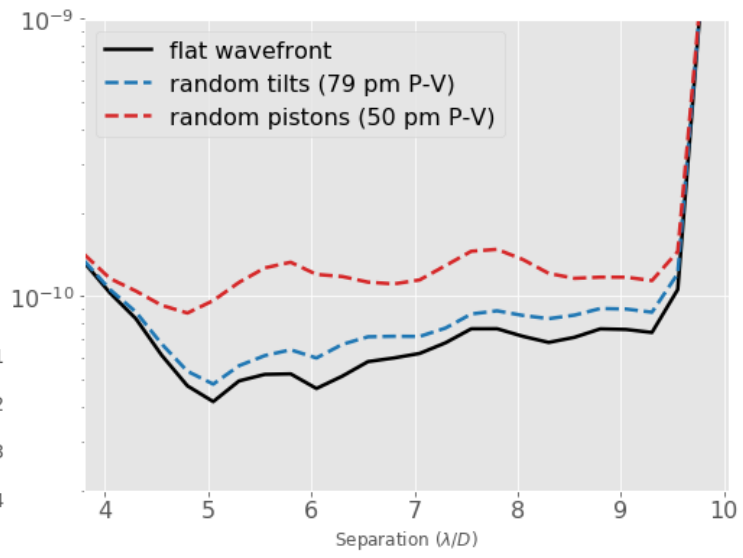

Figure 11: Sensitivity of the APLC design to segment level tip-tilt and piston. Courtesy of Roser Juanola-Parramon

instrument (along with the telescope) feature enough degrees of freedom to stabilize the wavefront at levels required for stable $\sim 10^{-10}$ contrast. 


\subsubsection{Observability: high heritage wavefront sensing techniques}

Even if the instrument has enough degrees of freedom to control for time varying wavefront disturbances, their observability (e.g. the ability to sense the relevant modes of the wavefront) need to be established. To do so we first rely on two optical diagnostics which have been developed over the past decade for high-contrast imaging:

- DH algorithms use the starlight in the dark hole to measure the wavelength dependent electrical field in the plane of the science camera, and use this information to calculate DM settings that create a DH in the final focal plane of the coronagraph. ${ }^{46-49}$ Note that in the absence of an IFS, a set of narrow band filters is required, which leads to a more complex filter wheel mechanism and larger overheads associated with wavefront control (since the multi-wavelength estimates need to be obtained sequentially). In this case, the timescales associated with observable wavefront disturbances can only be larger than the exposure times necessary to obtain sufficient SNR on the electrical field associated with $\sim 10^{-10}$ speckles, at all the sensed wavelengths. Indeed, noisy speckle estimates would yield irrelevant wavefront correction commands. In the case of the LUVOIR architecture "A" these can be of the order of hours. Even if such timescales can somewhat be lowered using predictive control, ${ }^{50,51}$ they will never be lower than the exposure necessary to observe an exo-earth at sufficient SNR. Note however that in spite of these temporal limitations, because of the presence of non common path error in the optical path of any other wavefront sensor, this is the only technique that provides and absolute wavefront measurements. It will thus be used to set the DH operating point of the wavefront control system.

- Low Order Wavefront Sensors use the starlight rejected by the coronagraph to correct for disturbance which are faster than the science exposure time. This was first demonstrated from the ground for low order aberrations, ${ }^{52-54}$ and is the strategy currently being matured for WFIRST. ${ }^{10}$ Each channel of the LUVOIR Architecture "A" coronagraph is equipped with Zernike based ${ }^{55-57}$ sensor. The architecture of each LOWFS follows the WFIRST implementation, ${ }^{10}$ using, for each of these sensing cameras, a detector technology similar than the one of the channel's science instrument, albeit with a lower pixel count (and not cooled to $70 \mathrm{~K}$ in the IR channel). This will enable wavefront sensing of low order modes on timescales of the order of a few seconds to a minute, depending on the brightness of the observed star.

\subsubsection{Observability: "novel" wavefront sensing techniques}

Because the starlight rejected by the coronagraph corresponds broadly to low spatial frequencies (again, if the coronagraph rejected high spatial frequencies, then the planet throughput would be low), these two aforementioned methods will be blind to mid-to-high spatial frequency variations faster than one (or a few) science exposure. Moreover the sensing timescale of these techniques is intrinsically limited by the brightness of the host star, and as a consequence performances will depend on the distance/temperature of the host star. As a result, the LUVOIR Architecture A features, in addition to the aforementioned concepts, two "novel" (e.g. non-WFIRST like) wavefront sensing strategies:

- In order to sense the high order modes that vary faster than one (or more) science exposure, each channel is equipped with a Zernike phase mask in the focal plane wheel of the coronagraph. During regular science operations, the three channels will operated simultaneously: however one of them will be used as an "out of band" wavefront sensor (e.g. not for science). In that case the focal plane wheel of the "out of band" channel will be a Zernike phase mask and the imaging detector will be used as a wavefront sensor. Because of chromatic non-common path errors this will not provide an absolute measure of the wavefront (we are assuming that this absolute measure is provided by the science channel, using the DH algorithms). Nevertheless this "out of band" sensor will provide rapid estimates of the mid-spatial frequency content of the wavefront variations (similar timescale as the LOWFS) and thus enable corrections using the DMs of the other two channels (operating in science mode). Algorithms for out of DH sensing (either spatially or spectrally) have been recently studied, ${ }^{58,59}$ and can be generalized to be used in conjunction with the Zernike sensor with segmented telescopes. ${ }^{60}$ Numerical simulations and experimental demonstrations of this are currently underway. ${ }^{61}$ 
- Finally, due to the segmented nature of the primary mirror, mechanical vibrations might induce wavefront changes that cannot be measured fast enough using diagnostics solely based on the photons of the target star (e.g at timescales smaller than a few second to a minute). As a consequence the primary mirror is equipped with capacitive edge sensors whose telemetry will be directly used feed the primary PZT adjustment actuators at fast frequency ${ }^{3}$ (a few ten of hertz). Note again that this fast closed loop will be not be absolute, it will be a relative correction based on the state of the primary when the DH algorithm shaped the coronagraph PSF. Ongoing work is being carried out to demonstrate the closed loop operations of capacitive segment edge sensing along with JWST like actuators equipped with a picometer resolution PZT fine stage. ${ }^{3}$

\subsubsection{System level considerations}

This wavefront sensing and control architecture relies on four different type of sensors (edge sensors, out of band sensor, LOWFS, DH electrical field estimate at science camera), spread of three different channels $(+$ the primary mirror), sensing commands to six different DMs spread over three channels (+ primary mirror), in order to correct wavefront changes along three broad time scales (few hours, seconds to minutes, less than a second). While the system complexity can seem daunting at first, we argue here that WFIRST will bring to TRL9 very fundamental aspects associated with operating nested wavefront control loops in space. Indeed, WFIRST will demonstrate closed loop operations of a LOFWS used in conjunction with a 2 DMs DH algorithm (albeit at contrast more modest than the ones enviousness by LUVOIR). By doing so, it will establish key subsystem level procedures necessary for LUVOIR such as the operations of the LOWFS while the science camera is being used to dig the $\mathrm{DH}$, command off-loads between in order to avoid actuators non linearity or to prevent loosing lock in one of the loops. In parallel, the out of band wavefront sensing and edge sensor technologies will be advanced. Thus in the post WFIRST era the technology associated to each one of the four wavefront sensing concepts discussed above will be matured, and simultaneous operations of two of them on a space based observatory will have been established. The remaining effort will consist on system level demonstrations of four nested loops operating in conjunction with one another. We encourage the community to get a head start for such demonstration and start now.

\section{CONCLUSION AND FUTURE WORK}

In this paper we have presented the coronagraph instrument for the LUVOIR Architecture "A". Throughout the manuscript we described the trades we made during the design process. Because the vocation of this instrument is to provide a baseline for the most ambitious earth-like finding instrument that could be possibly launched into the 2030's, we have designed an complex system that meets the ambitious science goals our was chartered by the LUVOIR STDT. Our next immediate step is now to finish assessing the yield of our designs via a full Design Reference Mission, in order to quantify the pertinence of our choice from a scientific standpoint (and adjust them if necessary). We also tried to privilege a number of technologies that will be matured by the WFIRST coronagraph instruments: in particular we baselined coronagraph masks, wavefront sensing and control subsystems that will be brought to TRL9 by WFIRST. System level demonstration of a coronagraph operating with the four nested picometer wavefront sensing and control loops described in this paper on a segmented aperture is the most critical technological aspect that needs to be advanced in the near future.

\section{ACKNOWLEDGMENTS}

This material is based upon work carried out under subcontracts No.1496556 and No. 1539872 with the Jet Propulsion Laboratory funded by NASA and administered by the California Institute of Technology.

\section{REFERENCES}

[1] Bolcar, M. R., Aloezos, S., Bly, V. T., Collins, C., Crooke, J., Dressing, C. D., Fantano, L., Feinberg, L. D., France, K., Gochar, G., Gong, Q., Hylan, J. E., Jones, A., Linares, I., Postman, M., Pueyo, L., Roberge, A., Sacks, L., Tompkins, S., and West, G., "The large uv/optical/infrared surveyor (luvoir): Decadal mission concept design update," SPIE proceedings 10398 (2017). 
[2] Acton, D. S., Knight, J. S., Contos, A., Grimaldi, S., Terry, J., Lightsey, P., Barto, A., League, B., Dean, B., Smith, J. S., Bowers, C., Aronstein, D., Feinberg, L., Hayden, W., Comeau, T., Soummer, R., Elliott, E., Perrin, M., and Starr, C. W., "Wavefront sensing and controls for the James Webb Space Telescope," in [Space Telescopes and Instrumentation 2012: Optical, Infrared, and Millimeter Wave], Society of PhotoOptical Instrumentation Engineers (SPIE) Conference Series 8442, 84422H (Sept. 2012).

[3] L. D. Feinberg, e. a., "Ultra-stable segmented telescope sensing and control architecture," Proc. SPIE 10398 (2017).

[4] Stark, C. C., Roberge, A., Mandell, A., Clampin, M., Domagal-Goldman, S. D., McElwain, M. W., and Stapelfeldt, K. R., "Lower Limits on Aperture Size for an ExoEarth Detecting Coronagraphic Mission," The Astrophysical Journal 808, 149 (Aug. 2015).

[5] Ruane, G., Jewell, J., Mawet, D., Pueyo, L., and Shaklan, S., "Apodized vortex coronagraph designs for segmented aperture telescopes," in [Proceedings of the SPIE], Proceedings of the SPIE 9912, 99122L99122L-13 (2016).

[6] Stark, C. C., Roberge, A., Mandell, A., and Robinson, T. D., "Maximizing the ExoEarth Candidate Yield from a Future Direct Imaging Mission," Astrophysical Journal 795, 122 (Nov. 2014).

[7] Arney, G. N., Meadows, V. S., Domagal-Goldman, S. D., Deming, D., Robinson, T. D., Tovar, G., Wolf, E. T., and Schwieterman, E., "Pale Orange Dots: The Impact of Organic Haze on the Habitability and Detectability of Earthlike Exoplanets," Astrophysical Journal 836, 49 (Feb. 2017).

[8] Robinson, T. D., "Characterizing Exoplanets for Habitability," ArXiv e-prints (Jan. 2017).

[9] Krist, J., Nemati, B., and Mennesson, B., "Numerical modeling of the proposed WFIRST-AFTA coronagraphs and their predicted performances," Journal of Astronomical Telescopes, Instruments, and Systems $\mathbf{2}$, 011003 (Jan. 2016).

[10] Shi, F., Balasubramanian, K., Bartos, R., Hein, R., Lam, R., Mandic, M., Moore, D., Moore, J., Patterson, K., Poberezhskiy, I., Shields, J., Sidick, E., Tang, H., Truong, T., Wallace, J. K., Wang, X., and Wilson, D. W., "Low order wavefront sensing and control for WFIRST coronagraph," in [Space Telescopes and Instrumentation 2016: Optical, Infrared, and Millimeter Wave], Society of Photo-Optical Instrumentation Engineers (SPIE) Conference Series 9904, 990418 (July 2016).

[11] Shaklan, S. B. and Green, J. J., "Reflectivity and optical surface height requirements in a broadband coronagraph. 1.Contrast floor due to controllable spatial frequencies," Applied Optics 45, 5143-5153 (July 2006).

[12] Cady, E., Prada, C. M., An, X., Balasubramanian, K., Diaz, R., Kasdin, N. J., Kern, B., Kuhnert, A., Nemati, B., Poberezhskiy, I., Eldorado Riggs, A. J., Zimmer, R., and Zimmerman, N., "Demonstration of high contrast with an obscured aperture with the WFIRST-AFTA shaped pupil coronagraph," Journal of Astronomical Telescopes, Instruments, and Systems 2, 011004 (Jan. 2016).

[13] S. C. Park, e. a., "Luvoir backplane thermal architecture development through the composite cte sensitivity study," Proc. SPIE 10398 (2017).

[14] Nemati, B., Effinger, R., Demers, R., Harding, L., Morrissey, P., Bush, N., Hall, D., and Skottfelt, J., "The effect of radiation-induced traps on the WFIRST coronagraph detectors," in [High Energy, Optical, and Infrared Detectors for Astronomy VII], Society of Photo-Optical Instrumentation Engineers (SPIE) Conference Series 9915, 99150M (Aug. 2016).

[15] Nemati, B., "Detector selection for the WFIRST-AFTA coronagraph integral field spectrograph," in [Space Telescopes and Instrumentation 2014: Optical, Infrared, and Millimeter Wave], Society of Photo-Optical Instrumentation Engineers (SPIE) Conference Series 9143, 91430Q (Aug. 2014).

[16] Macintosh, B. A., Graham, J. R., Palmer, D. W., Doyon, R., Dunn, J., Gavel, D. T., Larkin, J., Oppenheimer, B., Saddlemyer, L., Sivaramakrishnan, A., Wallace, J. K., Bauman, B., Erickson, D. A., Marois, C., Poyneer, L. A., and Soummer, R., "The Gemini Planet Imager: from science to design to construction," in [Proceedings of the SPIE], Proceedings of the SPIE 7015, 701518 (July 2008).

[17] Beuzit, J.-L., Feldt, M., Mouillet, D., Dohlen, K., Puget, P., Wildi, F., and SPHERE Consortium, "SPHERE: a planet imager for the VLT," in [Proceedings of the conference In the Spirit of Lyot 2010], Proceedings of the conference In the Spirit of Lyot 2010, 44 (Oct. 2010). 
[18] Hinkley, S., Oppenheimer, B. R., Zimmerman, N., Brenner, D., Parry, I. R., Crepp, J. R., Vasisht, G., Ligon, E., King, D., and Soummer, R., "A new high contrast imaging program at Palomar observatory," Publications of the Astronomical Society of the Pacific 123(899), 74-86 (2011).

[19] Groff, T. D., Chilcote, J., Kasdin, N. J., Galvin, M., Loomis, C., Carr, M. A., Brandt, T., Knapp, G., Limbach, M. A., Guyon, O., Jovanovic, N., McElwain, M. W., Takato, N., and Hayashi, M., "Laboratory testing and performance verification of the CHARIS integral field spectrograph," in [Ground-based and Airborne Instrumentation for Astronomy VI], Society of Photo-Optical Instrumentation Engineers (SPIE) Conference Series 9908, 99080 O (Aug. 2016).

[20] Demers, R. T., Dekens, F., Calvet, R., Chang, Z., Effinger, R., Ek, E., Hovland, L., Jones, L., Loc, A., Nemati, B., Noecker, C., Neville, T., Pham, H., Rud, M., Tang, H., and Villalvazo, J., "Requirements and design reference mission for the WFIRST/AFTA coronagraph instrument," in [Techniques and Instrumentation for Detection of Exoplanets VII], Society of Photo-Optical Instrumentation Engineers (SPIE) Conference Series 9605, 960502 (Sept. 2015).

[21] Mawet, D., Ruane, G., Xuan, W., Echeverri, D., Klimovich, N., Randolph, M., Fucik, J., Wallace, J. K., Wang, J., Vasisht, G., Dekany, R., Mennesson, B., Choquet, E., Delorme, J.-R., and Serabyn, E., "Observing Exoplanets with High-dispersion Coronagraphy. II. Demonstration of an Active Single-mode Fiber Injection Unit," Astrophysical Journal 838, 92 (Apr. 2017).

[22] Snellen, I. A. G., Brandl, B. R., de Kok, R. J., Brogi, M., Birkby, J., and Schwarz, H., "Fast spin of the young extrasolar planet $\beta$ Pictoris b," Nature 509, 63-65 (May 2014).

[23] Wang, J., Mawet, D., Ruane, G., Hu, R., and Benneke, B., "Observing Exoplanets with High Dispersion Coronagraphy. I. The Scientific Potential of Current and Next-generation Large Ground and Space Telescopes," Astronomical Journal 153, 183 (Apr. 2017).

[24] Snellen, I., de Kok, R., Birkby, J. L., Brandl, B., Brogi, M., Keller, C., Kenworthy, M., Schwarz, H., and Stuik, R., "Combining high-dispersion spectroscopy with high contrast imaging: Probing rocky planets around our nearest neighbors," Astronomy and Astrophysics 576, A59 (Apr. 2015).

[25] Lovis, C., Snellen, I., Mouillet, D., Pepe, F., Wildi, F., Astudillo-Defru, N., Beuzit, J.-L., Bonfils, X., Cheetham, A., Conod, U., Delfosse, X., Ehrenreich, D., Figueira, P., Forveille, T., Martins, J. H. C., Quanz, S. P., Santos, N. C., Schmid, H.-M., Ségransan, D., and Udry, S., "Atmospheric characterization of Proxima b by coupling the SPHERE high-contrast imager to the ESPRESSO spectrograph," Astronomy and Astrophysics 599, A16 (Mar. 2017).

[26] Breckinridge, J. B. and Lillie, C. F., "Prime focus architectures for large space telescopes: reduce surfaces to save cost," in [Space Telescopes and Instrumentation 2016: Optical, Infrared, and Millimeter Wave], Society of Photo-Optical Instrumentation Engineers (SPIE) Conference Series 9904, 99044K (July 2016).

[27] Bifano, T. G., Bierden, P. A., Zhu, H., Cornelissen, S., and Kim, J. H., "Megapixel wavefront correctors," in [Advancements in Adaptive Optics], Bonaccini Calia, D., Ellerbroek, B. L., and Ragazzoni, R., eds., Society of Photo-Optical Instrumentation Engineers (SPIE) Conference Series 5490, 1472-1481 (Oct. 2004).

[28] Pueyo, L. and Norman, C., "High-contrast Imaging with an Arbitrary Aperture: Active Compensation of Aperture Discontinuities," The Astrophysical Journal 769, 102 (June 2013).

[29] Cahoy, K., Marinan, A., Kerr, C., Cheng, K., and Jamil, S., "CubeSat deformable mirror demonstration," in [Space Telescopes and Instrumentation 2012: Optical, Infrared, and Millimeter Wave], Society of PhotoOptical Instrumentation Engineers (SPIE) Conference Series 8442, 84424F (Sept. 2012).

[30] Marinan, A., Cahoy, K., Webber, M., Belikov, R., and Bendek, E., "Payload characterization for CubeSat demonstration of MEMS deformable mirrors," in [Adaptive Optics Systems IV], Society of Photo-Optical Instrumentation Engineers (SPIE) Conference Series 9148, $91483 Z$ (Aug. 2014).

[31] Soummer, R., Aime, C., and Falloon, P. E., "Stellar coronagraphy with prolate apodized circular apertures," Astronomy and Astrophysics 397, 1161-1172 (Jan. 2003).

[32] N'Diaye, M., Soummer, R., Pueyo, L., Carlotti, A., Stark, C. C., and Perrin, M. D., "Apodized Pupil Lyot Coronagraphs for Arbitrary Apertures. V. Hybrid Shaped Pupil Designs for Imaging Earth-like planets with Future Space Observatories," The Astrophysical Journal 818, 163 (Feb. 2016).

[33] Zimmerman, N. T., Eldorado Riggs, A. J., Jeremy Kasdin, N., Carlotti, A., and Vanderbei, R. J., "Shaped pupil Lyot coronagraphs: high-contrast solutions for restricted focal planes," Journal of Astronomical Telescopes, Instruments, and Systems 2, 011012 (Jan. 2016). 
[34] Mawet, D., Serabyn, E., Liewer, K., Hanot, C., McEldowney, S., Shemo, D., and O’Brien, N., "Optical Vectorial Vortex Coronagraphs using Liquid Crystal Polymers: theory, manufacturing and laboratory demonstration," Optics Express 17, 1902-1918 (Feb. 2009).

[35] Mawet, D., Serabyn, E., Moody, D., Kern, B., Niessner, A., Kuhnert, A., Shemo, D., Chipman, R., McClain, S., and Trauger, J., "Recent results of the second generation of vector vortex coronagraphs on the highcontrast imaging testbed at JPL," in [Proceedings of the SPIE], Proceedings of the SPIE 8151, 81511D (Oct. 2011).

[36] Riggs, A. J. E., Zimmerman, N., Carlotti, A., Kasdin, N. J., and Vanderbei, R., "Shaped pupil design for future space telescopes," in [Space Telescopes and Instrumentation 2014: Optical, Infrared, and Millimeter Wave], Society of Photo-Optical Instrumentation Engineers (SPIE) Conference Series 9143, 914325 (Aug. 2014).

[37] N'Diaye, M., Pueyo, L., and Soummer, R., "Apodized Pupil Lyot Coronagraphs for Arbitrary Apertures. IV. Reduced Inner Working Angle and Increased Robustness to Low-order Aberrations," The Astrophysical Journal 799, 225 (Feb. 2015).

[38] Mawet, D., Pueyo, L., Carlotti, A., Mennesson, B., Serabyn, E., and Wallace, J. K., "Ring-apodized Vortex Coronagraphs for Obscured Telescopes. I. Transmissive Ring Apodizers," The Astrophysical Journal Supplement Series 209, 7 (Nov. 2013).

[39] Fogarty, K., Pueyo, L., Mazoyer, J., and N'Diaye, M., "Polynomial Apodizers for Centrally Obscured Vortex Coronagraphs," ArXiv e-prints (Mar. 2017).

[40] Mazoyer, J., Pueyo, L., N'Diaye, M., Fogarty, K., Zimmerman, N., Soummer, R., Shaklan, and Norman, C., "Active correction of aperture discontinuities - optimized stroke minimization I: a new adaptive interaction matrix algorithm," submitted to AJ (2017).

[41] Mazoyer, J., Pueyo, L., N'Diaye, M., Fogarty, K., Zimmerman, N., Soummer, R., Shaklan, and Norman, C., "Active correction of aperture discontinuities - optimized stroke minimization I: a new adaptive control matrix algorithm," submitted to AJ (2017).

[42] Ruane, G., Mawet, D., Jewell, J., and Shaklan, S., "Performance and sensitivity of vortex coronagraphs on segmented space telescopes," ArXiv e-prints (Aug. 2017).

[43] Garrett, D., Savransky, D., and Macintosh, B., "A Simple Depth-of-Search Metric for Exoplanet Imaging Surveys," Astronomical Journal 154, 47 (Aug. 2017).

[44] Savransky, D. and Garrett, D., "WFIRST-AFTA coronagraph science yield modeling with EXOSIMS," Journal of Astronomical Telescopes, Instruments, and Systems 2, 011006 (Jan. 2016).

[45] Pueyo, L. and Kasdin, N. J., "Polychromatic Compensation of Propagated Aberrations for High-Contrast Imaging," The Astrophysical Journal 666, 609-625 (Sept. 2007).

[46] Trauger, J. T. and Traub, W. A., "A laboratory demonstration of the capability to image an Earth-like extrasolar planet," Nature 446, 771-773 (Apr. 2007).

[47] Pueyo, L., Kay, J., Kasdin, N. J., Groff, T., McElwain, M., Give'on, A., and Belikov, R., "Optimal dark hole generation via two deformable mirrors with stroke minimization," Applied Optics 48, 6296 (Nov. 2009).

[48] Bordé, P. J. and Traub, W. A., "High-Contrast Imaging from Space: Speckle Nulling in a Low-Aberration Regime," The Astrophysical Journal 638, 488 (Feb. 2006).

[49] Give'on, A., Kern, B., Shaklan, S., Moody, D. C., and Pueyo, L., "Broadband wavefront correction algorithm for high-contrast imaging systems," in [Proceedings of the SPIE], Proceedings of the SPIE 6691, 66910A (Sept. 2007).

[50] Groff, T. D. and Jeremy Kasdin, N., "Kalman filtering techniques for focal plane electric field estimation," Journal of the Optical Society of America A 30, 128 (Jan. 2013).

[51] Riggs, A. J. E., Kasdin, N. J., and Groff, T. D., "Wavefront correction with Kalman filtering for the WFIRST-AFTA coronagraph instrument," in [Proceedings of the SPIE], Proceedings of the SPIE 9605, 960507 (Sept. 2015).

[52] Lloyd, J. P. and Sivaramakrishnan, A., "Tip-Tilt Error in Lyot Coronagraphs," Astrophysical Journal 621, 1153-1158 (Mar. 2005). 
[53] Guyon, O., Matsuo, T., and Angel, R., "Coronagraphic Low-Order Wave-Front Sensor: Principle and Application to a Phase-Induced Amplitude Coronagraph," The Astrophysical Journal 693, 75-84 (Mar. 2009).

[54] Singh, G., Lozi, J., Guyon, O., Baudoz, P., Jovanovic, N., Martinache, F., Kudo, T., Serabyn, E., and Kuhn, J., "On-Sky Demonstration of Low-Order Wavefront Sensing and Control with Focal Plane Phase Mask Coronagraphs," Publications of the Astronomical Society of the Pacific, 127, 857 (Sept. 2015).

[55] Wallace, J. K., Crawford, S., Loya, F., and Moore, J., "A phase-shifting Zernike wavefront sensor for the Palomar P3k adaptive optics system," in [Proceedings of the SPIE], Proceedings of the SPIE 8447, 84472K (July 2012).

[56] N'Diaye, M., Dohlen, K., Fusco, T., and Paul, B., "Calibration of quasi-static aberrations in exoplanet direct-imaging instruments with a Zernike phase-mask sensor," Astronomy and Astrophysics 555, A94 (July 2013).

[57] N'Diaye, M., Vigan, A., Dohlen, K., Sauvage, J.-F., Caillat, A., Costille, A., Girard, J. H. V., Beuzit, J.-L., Fusco, T., Blanchard, P., Le Merrer, J., Le Mignant, D., Madec, F., Moreaux, G., Mouillet, D., Puget, P., and Zins, G., "Calibration of quasi-static aberrations in exoplanet direct-imaging instruments with a Zernike phase-mask sensor. II. Concept validation with ZELDA on VLT/SPHERE," Astronomy and Astrophysics 592, A79 (Aug. 2016).

[58] Guyon, O., Miller, K., Males, J., Belikov, R., and Kern, B., "Spectral Linear Dark Field Control: Stabilizing Deep Contrast for Exoplanet Imaging Using out-of-band Speckle Field," ArXiv e-prints (June 2017).

[59] Miller, K., Guyon, O., and Males, J. R., "Spatial Linear Dark Field Control: Stabilizing Deep Contrast for Exoplanet Imaging Using Bright Speckles," ArXiv e-prints (Mar. 2017).

[60] Janin-Potiron, P., N'Diaye, M., Martinez, P., Vigan, A., Dohlen, K., and Carbillet, M., "Fine cophasing of segmented aperture telescopes with ZELDA, a Zernike wavefront sensor in the diffraction-limited regime," Astronomy and Astrophysics 603, A23 (July 2017).

[61] Jackson, K., Wallace, J. K., and Pellegrino, S., "Co-phasing primary mirror segments of an optical space telescope using a long stroke Zernike WFS," in [Space Telescopes and Instrumentation 2016: Optical, Infrared, and Millimeter Wave], Society of Photo-Optical Instrumentation Engineers (SPIE) Conference Series 9904, 99046D (July 2016). 